\title{
The Association between Job Related Factors, Short Sleep and Obesity
}

\author{
Lee DI MILIA ${ }^{1,2 *}$ and Kerry MUMMERY ${ }^{3}$
}

\author{
${ }^{1}$ School of Management and the Institute for Health and Social Sciences, Central Queensland University, Bruce \\ Highway Rockhampton, QLD 4702, Australia \\ ${ }^{2}$ Department of Psychology, University of Connecticut, Babbidge Road Storrs, CT 06269, USA \\ ${ }^{3}$ School of Health and Human Performance and the Institute for Health and Social Sciences, Central Queensland \\ University, QLD 4701, Australia
}

Received August 5, 2008 and accepted January 27, 2009

\begin{abstract}
Epidemiological studies have found a consistent inverse relationship between increased body mass index (BMI) and sleep duration. These studies have not controlled for the role that job related factors such as shift work and working hours may have on this relationship. A cross sectional survey of shift and day workers $(\mathrm{N}=346 ; 292$ males and 59 females) was employed to investigate the association between obesity, job related factors and sleep duration. Mean age for the overall sample was $41.1 \mathrm{yr}( \pm 11.1)$. Mean BMI was significantly higher in shift workers than in day workers $(p<.001)$. Mean BMI $(12.60 \mathrm{~h} \pm 0.41)$ was also significantly $(p<.001)$ higher in the group working long daily hours followed by medium working hours $(10.95 \mathrm{~h} \pm 0.56)$ and short working hours $(8.72 \mathrm{~h} \pm 0.56)$. Obese individuals worked significantly longer hours $(p<.001)$ and slept 18 min less per day $(p<.06)$ compared to those with a normal BMI. The adjusted odds ratio (OR) indicated the most significant predictor of obesity was long working hours $(\mathrm{OR}=2.82$, CI: 1.10-7.19), followed by being older $(O R=2.05$, CI: $1.17-3.59)$ and short sleep duration $(O R=1.92$, CI: 1.03-3.55). The limitations of the study are discussed.
\end{abstract}

Key words: Age, Obesity, Shift work, Sleep, Work hours

\section{Introduction}

The relationship between work schedules and long working hours on health has been the subject of much research over several decades. Recent reviews of this literature can be found elsewhere ${ }^{1-3}$. One of the strongest findings across the literature is the inverse relationship between night work, long work hours and sleep. A number of studies have linked sleep loss with a number of impairments including increased reaction time and reduced cognitive and driving performance ${ }^{4-6)}$. More recently a number of studies have implicated short sleep duration (eg. five to seven hours) with greater body mass index (BMI) in child ${ }^{7)}$ and adult populations ${ }^{8-10)}$. A number of these latter studies are large scale epidemiological studies and while valuable, they lack information on the type of working arrangements in the sample. Therefore,

*To whom correspondence should be addressed. it is unclear from these studies whether sleep is the major link with BMI or whether job related factors such as shift work or working hours are also implicated in this relationship.

There are relatively few studies that have examined the role of work schedules and working hours on BMI. Di Lorenzo et al. ${ }^{11)}$ reported BMI was significantly greater among rotating shift workers ( $8 \mathrm{~h}$ shifts) compared to day workers. Biggi et al. ${ }^{12)}$ found permanent night workers (6 h shifts) had a greater BMI compared to day workers. In a study involving $12 \mathrm{~h}$ shift workers Parkes ${ }^{13)}$ concluded that continued exposure to shiftwork increased BMI over and above the impact of age alone when compared to day workers. van Amelsvoort et al. ${ }^{14)}$ also reported that after adjusting for age, shift workers had higher BMI but did not report the duration of the shift. Similarly, in a large study of truck drivers on irregular work schedules, Moreno et al. ${ }^{15)}$ reported $28 \%$ of drivers were obese $\left(B M I \geq 30 \mathrm{~kg} / \mathrm{m}^{2}\right.$ ). The odds ratio result indi- 
cated an association between obesity, sleeping less than $8 \mathrm{~h}$ per day, aged over $40 \mathrm{yr}$, hypertension and snoring.

Independent of work schedules, there is some evidence that long work hours are also linked to BMI although the results are not consistent ${ }^{2}$. Using a large Asian random sample, Ko et al. ${ }^{16)}$ recently addressed the issue of daily working hours and obesity. In addition to finding the inverse relationship between BMI and short sleep $(<6 \mathrm{~h})$, they reported BMI was greater in participants with a mean daily working time of $11 \mathrm{~h}( \pm 1.38)$. Long work hours may directly reduce time for physical activity since it competes with other demands and perhaps indirectly via fatigue, making exercise less attractive ${ }^{17)}$. A further complication that may mediate the relationship between work and BMI is the nature of the daily work demands. For example, Mummery et al. ${ }^{18)}$ concluded sedentary white collar employees were more likely to be obese than blue collar employees. The physical nature of some blue collar work may result in sufficient energy expenditure to maintain a normal BMI.

We have argued there is some evidence linking shift work and long work hours with BMI. In addition, a number of epidemiological studies ${ }^{8-10)}$ have implicated short sleep duration as a major factor in higher levels of BMI. However, it is unclear which of these factors is more important in explaining obesity. The aim of this pilot study is to use logistic regression to simultaneously examine the contribution of shift work, work hours and sleep duration on obesity. We focus on obesity since it is reaching epidemic proportions. Australian estimates suggest some $29 \%$ of the population will be obese by $2025^{19}$.

\section{Participants and Methods}

A cross sectional survey was administered to 804 Australian participants employed in the coal industry and 275 participants from a regional university. These samples were chosen to obtain a range of working hours from white and blue collar employees. Replies were received from 201 shift workers (SW) and 71 participants employed as day workers (DW) from the coal industry; response rate of $34 \%$. A total of 74 replies were received from the university employees $(27 \%)$. The SW worked two day shifts followed by one day off, two night shifts and then four days off. The day and night shifts were approximately $12.6 \mathrm{~h}$ in duration per shift. The two DW samples commenced work between 07:00 and 08:30 from Monday to Friday.

Participants received a package that included an information sheet, the survey and a self-addressed reply envelope. The survey collected a range of biographical details, sleep duration, work schedules and daily working hours per shift. The study met the requirements of the human research ethics committee at Central Queensland University.

\section{Data Analyses Strategy}

A number of categories were created based on the responses to the survey. The participant's mass and height $\left(\mathrm{kg} / \mathrm{m}^{2}\right)$ were used to calculate BMI using criteria obtained from the National Heart Lung and Blood Institute ${ }^{20)}$ : 'normal' $\left(<25 \mathrm{~kg} / \mathrm{m}^{2}\right)$, 'overweight' $\left(25-29.9 \mathrm{~kg} / \mathrm{m}^{2}\right)$ and 'obese' $\left(\geq 30 \mathrm{~kg} / \mathrm{m}^{2}\right)$.

In keeping with other studies ${ }^{15)}$ we created two age groups using a median split (41 yr). The mean age for the young group was $31.61 \mathrm{yr}( \pm 6.45)$ and $50.15 \mathrm{yr}$ $( \pm 5.64)$ in the old group. Participants were allocated into the three groups based on the mean work duration per shift; 'short' $(\mathrm{M}=8.72 \mathrm{~h} \pm 0.56), \quad$ 'medium' $(\mathrm{M}=10.95 \mathrm{~h} \pm 0.56)$ and 'long' $(\mathrm{M}=12.60 \mathrm{~h} \pm 0.41)$. To a large extent the long hours were worked by SW, the medium hours by DW employed by the mines and the short hours by DW at the university; Spearman's rho $=0.82$ $(p<.001)$.

Sleep duration was calculated using the mean from two sleep items; "how much sleep do you need between day shifts" and "how much sleep do you need between days off". We used the median split $(8.00 \mathrm{~h})$ to create a 'short' $(\mathrm{M}=7.36 \mathrm{~h} \pm 0.66)$ and 'long' $(\mathrm{M}=8.85 \mathrm{~h} \pm 0.53)$ sleep group $(p<.001)$ in order to examine the link between sleep duration and BMI. We asked participants if they snored and categorised them accordingly. The large majority of participants $(91 \%)$ reported being married or partnered and this provided some assurance that these responses were valid. The SW and DW made up the work schedule category. In addition, the SW were considered bluecollar workers as the work required heavy physical lifting and regular walking. The two DW samples were considered white-collar since they were employed as professionals, supervisors, academics and administrative staff.

The $\chi^{2}$ test was used to test for differences between categorical data and one-way ANOVA was used to examine for mean differences. Logistic regression was employed to examine the association between obesity and the predictor variables; sleep length, age, daily work hours, work schedule and gender.

\section{Results}

The overall sample was predominantly male (83\%) and they mostly worked medium to long work hours. The mean working hours per shift for males was $11.76 \mathrm{~h}$ $( \pm 1.41)$ and $9.70 \mathrm{~h} \pm$ (1.60) for females. Mean BMI was significantly $(p<.001)$ higher in males $(\mathrm{M}=27.81 \pm 5.12)$ than females $(\mathrm{M}=24.81 \pm 3.79, p<.001)$. Mean age for 
Table 1. Distribution of categorical variables by daily work hours (\%)

\begin{tabular}{lcccc}
\hline \multicolumn{5}{c}{ Daily work hours } \\
\hline Variable & Short (N=74) & Medium (N=70) & Long (N=195) & $p$ \\
\hline Gender: Male & $45.9 \%$ & $88.6 \%$ & $94.9 \%$ & .001 \\
$\quad$ Female & $54.1 \%$ & $11.4 \%$ & $5.1 \%$ & \\
Age: Young & $39.2 \%$ & $47.1 \%$ & $52.8 \%$ & NS \\
$\quad$ Old & $60.8 \%$ & $52.9 \%$ & $47.2 \%$ & \\
BMI: Normal & $47.1 \%$ & $30.4 \%$ & $24.7 \%$ & \\
$\quad$ Overweight & $41.4 \%$ & $47.8 \%$ & $48.4 \%$ & .01 \\
$\quad$ Obese & $11.4 \%$ & $21.7 \%$ & $26.8 \%$ & \\
Snore: Yes & $41.1 \%$ & $52.9 \%$ & $65.6 \%$ & .001 \\
$\quad$ No & $58.9 \%$ & $47.1 \%$ & $34.4 \%$ & \\
WS: Shift work & $0.0 \%$ & $13.4 \%$ & $86.6 \%$ & .001 \\
$\quad$ Day work & $53.6 \%$ & $31.2 \%$ & $15.2 \%$ & \\
\hline
\end{tabular}

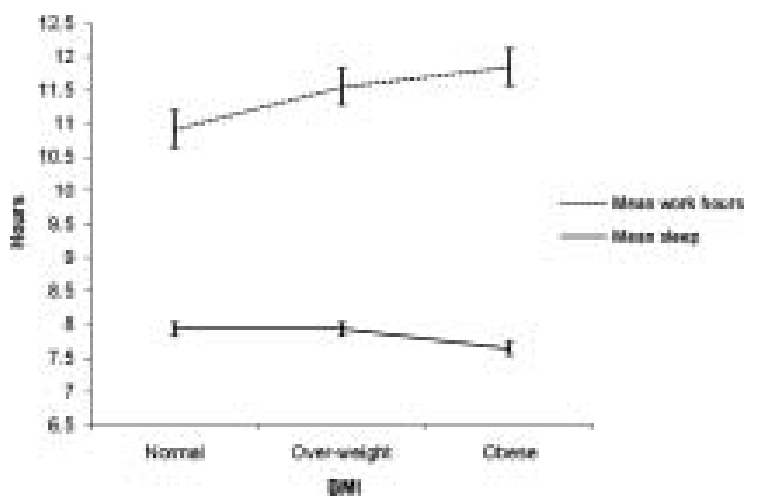

Fig. 1. Daily mean work hours and mean sleep duration by BMI categories.

SE bars are shown.

the total sample was $41.06 \mathrm{yr}(\mathrm{SD}=11.07)$ and did not significantly differ between the three work hour groups.

Mean BMI was significantly higher $(p<.001)$ in SW $(\mathrm{M}=28.10 \pm 5.43)$ than in $\mathrm{DW}(\mathrm{M}=26.19 \pm 4.38)$. Mean BMI was also was significantly higher $(p<.001)$ in the long hours group $(\mathrm{M}=28.14 \pm 5.56)$, less in the medium group $(27.37 \pm 4.43)$ and lowest in the short working hours group $(\mathrm{M}=25.27 \pm 4.06)$. The results from a series of $\chi^{2}$ tests indicated that the percentage of gender, BMI, snoring and work schedules were significantly different across the three work hour groups. Males, SW and snoring were more likely in the long working hours group. These results can be found in Table 1 .

The result obtained from a one-way ANOVA indicated the obese participants worked significantly longer hours $F(2,328=8.11 p<0.001)$, and slept $18 \mathrm{~min}$ less per day compared to participants with a normal BMI $(p<0.06)$. The means are shown in Fig. 1. In addition, mean sleep duration $(7.87 \pm .85)$ was non-significant between the daily work hour groups $(p<.10)$ but sleep duration increased as daily work hours increased.

The association between obesity and the predictor variables were examined via logistic regression. The Hosmer-Lemeshow inferential goodness-of-fit test was non-significant $(p>.05)$ indicating the model fitted the data well. The adjusted OR results suggested the older age group ( $\mathrm{OR}=2.06 ; \mathrm{CI}=1.17-3.62)$ and short sleep duration $(\mathrm{OR}=2.04 ; \mathrm{CI}=1.09-3.82)$ predicted obesity. Given the high correlation between daily work hours and work schedule (Spearman's rho $=0.82$ ) we repeated the logistic regression but excluded work schedule from the analysis. The Hosmer-Lemeshow inferential goodness-of-fit test remained non-significant. The adjusted OR confirmed the significant association with being older and short sleep. However, working long hours became significant and had the highest odds ratio $(\mathrm{OR}=2.82$; $\mathrm{CI}=1.10-7.19)$. The results from this analysis can be found in Table 2.

\section{Discussion}

The literature has independently assessed the role of job related factors and sleep duration as factors that explain obesity. The strength of this study is that it concurrently examined the contribution of these variables in predicting obesity.

Consistent with the literature we found that shift workers had a higher BMI ${ }^{1-14)}$. We had proposed that the physical nature of the shift workers jobs may have resulted in sufficient energy expenditure to lower their BMI but this was not supported. Not surprisingly, since the shift workers also worked longer daily hours, we found that long work hours were also linked to higher levels of BMI. Our results therefore, support the findings of Ko et al. ${ }^{16}$. This finding is important since they are obtained from two quite different cultures. Our results also demonstrated the 
Table 2. Crude and adjusted odds ratios* (OR) for variables associated with obesity

\begin{tabular}{lccccccc}
\hline Variable & Category & Crude OR & $95 \%$ CI & $p$ & Adjusted OR & $95 \%$ CI & $p$ \\
\hline Sleep length: & Short & 1.51 & $.85-2.69$ & .16 & 1.92 & $1.03-3.55$ & .05 \\
& Long & 1.00 & & & & & \\
Age: & Old & 1.98 & $1.16-3.35$ & .01 & 2.05 & $1.17-3.59$ & .05 \\
& Young & 1.00 & & & & & \\
Daily work hours: & Long & 2.84 & $1.27-6.35$ & .01 & 2.82 & $1.10-7.19$ & .05 \\
& Medium & 2.15 & $.85-5.47$ & .11 & 1.81 & $.64-5.10$ & .26 \\
& Short & 1.00 & & & & & \\
Gender: & Male & 3.32 & $1.28-8.65$ & .01 & 2.31 & $.77-6.90$ & .14 \\
& Female & 1.00 & & & & &
\end{tabular}

*Logistic regression simultaneously controls for the contribution of each predictor variable on obesity.

inverse relationship between BMI and sleep duration ${ }^{8-10)}$.

The key question in this study however, was whether shift work, long hours or short sleep duration best predicted obesity. We were unable to directly answer this question since shift work and long working hours were highly correlated. With this caveat, the odds ratio from our analyses indicated the strongest predictor of obesity was working long daily hours, followed by being older and then short sleep. There is some evidence that long working hours may be more problematic than shift work. In two studies of engineers on day work, Sasaki et al. ${ }^{21,22)}$ reported an inverse link between long working hours and sleep duration. Being older was also reported to be linked to obesity in other studies ${ }^{15)}$ and is most likely a result of reduced physical activity. Increasing age however, is also related to less sleep duration ${ }^{23)}$ and greater sleep disturbances ${ }^{24)}$.

The cross sectional design of this study prevents a causal argument to explain the findings. However, one possibility is that working long hours may interfere with a number of other activities such as sleep, exercise and meeting other family/social demands. Ko et al. ${ }^{16)}$ postulated that long hours and short sleep may be symptomatic of individuals under high levels of stress. Vgontzas et $a l .{ }^{25)}$ also reported that obese short sleepers had elevated levels of emotional stress. In turn, there is some evidence of increased food intake among stressed individuals ${ }^{26,27}$. A number of shift work and sleep studies ${ }^{11,12,28)}$ have proposed that increased BMI in shift workers may be the result of metabolic changes (eg. leptin and ghrelin) that result in mass gain. Another possibility is that short sleep may result in fatigue making exercise less attractive ${ }^{17}$. Indeed, some studies have concluded short sleepers are physically inactive ${ }^{8)}$, eat more fast food ${ }^{9)}$ and spend more time watching television ${ }^{29}$. Each of these explanations may not be mutually exclusive and future studies could usefully test these competing pathways.

Our results require replication and if supported, suggest two interventions to reduce obesity. The first is to limit working hours to some $11 \mathrm{~h}$ per shift (Fig. 1) in order to provide more time for sleep and non-work activity. Second, sleep extension may assist to lower BMI. Although just failing to be significant, individuals with a normal BMI slept an additional 18 min per day compared to obese individuals. Our sleep difference is similar to Vorona et al. $^{30)}$ who found a 20 min difference between normal and obese individuals.

There are some limitations to the study and therefore, some caution is required in interpreting the results. The sample comfortably met the observation to predictor ratio $(10: 1)^{31)}$ for the use of logistic regression, but it was still relatively small. For example, the long work hours were primarily worked by shift workers and thus we had too few shift workers working $8 \mathrm{~h}$ shifts. In addition, to allow a comparison of work schedules we pooled two different sets of DW. While the groups performed white-collar work, the coal industry participants worked about $1.5 \mathrm{~h}$ longer per day than the university employees. Thus the DW category was confounded by differences in type and duration of work. Second, the self-report protocol raises the possibility of bias resulting from common method variance. While there is evidence that self-reported mass may be underestimated, they are not considered significant enough to affect the results ${ }^{32}$. Self-reported sleep length and actigraphic sleep recordings have been shown to be well correlated ${ }^{33)}$ but it is the case that the accuracy depends on the cognitive and functional abilities of participants ${ }^{34)}$ Third, it may be the case that other confounders were present that we could not control (eg. energy intake and physical activity). We recommend future studies employ a stronger study design, make use of much larger samples, incorporate the use of objective measures and examine the role of other confounders.

\section{Acknowledgement}

This study was funded by Coal Services Australia Health and Safety Trust. 


\section{References}

1) Caruso CC (2006) Possible broad impacts of long work hours. Ind Health 44, 531-6.

2) van der Hulst M (2003) Long workhours and health. Scand J Work Environ Health 29, 171-88.

3) Tucker P (2006) Compressed working weeks. Conditions of work and employment series no. 12. International Labour Office, Geneva.

4) Otmani S, Pebayle T, Roget J, Muzet A (2005) Effect of driving duration and partial sleep deprivation on subsequent alertness and performance of car drivers. Physiol Beh 84, 715-24.

5) Philip P, Sagaspe P, Taillard J, Moore N, Guilleminault C, Sanchez-Ortuno M (2003) Fatigue, sleep restriction, and performance in automobile drivers: a controlled study in a natural environment. Sleep 26, 277-80.

6) Van Dongen HPA, Maislin G, Mullington JM, Dinges DF (2003) The cumulative cost of additional wakefulness: dose-response effects on neurobehavioral functions and sleep physiology from chronic sleep restriction and total sleep deprivation. Sleep 26, 117-26.

7) Chaput J-P, Brunet M, Tremblay A (2006) Relationship between short sleeping hours and childhood overweight/obesity: results from the 'Quebec en Forme' project. Int J Obes 31, 1080-5.

8) Fogelholm M, Kronholm E, Kukkonen-Harjula K, Partonen T, Harma M (2007) Sleep-related disturbances and physical inactivity are independently associated with obesity in adults. Int J Obes 31, 1713-21.

9) Stamatakis KA, Brwonson RC (2008) Sleep duration and obesity-related factors in the rural Midwest. Prev Med 46, 439-44.

10) Bjorvatn B, Sagen IM, Oyane N, Waage S, Fetveit A, Pallesen S, Ursin R (2007) The association between sleep duration, body mass index and metabolic measures in the Hordaland health study. J Sleep Res 16, 66-76.

11) Di Lorenzo L, De Pergola G, Zocchetti C, L'Abbate N, Basso A, Pannacciulli N, Cignarelli M, Girogino R, Soleo L (2003) Effect of shift work on body mass index: results of a study performed in 319 glucose-tolerant men working in a southern Italian industry. Int J Obes 27, 1353-8.

12) Biggi N, Consonni D, Galluzzo V, Sogliani M, Costa $\mathrm{G}$ (2008) Metabolic syndrome in permanent night workers. Chronobiol Int 25, 443-54.

13) Parkes KR (2002) Shift work and age as interactive predictors of body mass index among offshore workers. Scan J Work Environ Health 28, 64-71.

14) van Amelsvoort LGPM, Schouten EG, Kok FJ (1999) Duration od shiftwork related to body mass index and waist to hip ratio. Int $\mathrm{J}$ Obes 23, 973-8.

15) Moreno CRC, Louzada FM, Teixeira LR, Borges F, Lorenzi-Filho G (2006) Short sleep is associated with obesity among truck drivers. Chronobiol Int 23, 1295-303.

16) Ko GTC, Chan JCN, Chan AWY, Wong PTS, Hui SSC,
Tong SDY, Ng S-M, Chow F, Chan CLW (2007) Association between sleeping hours, working hours and obesity in Hong Kong chinese: the 'better health for better Hong Kong' health promotion campaign. Int J Obes 31, 254-60.

17) Fogelholm M, Kronholm E, Kukkonen-Harjula K, Partonen T, Harma M (2007) Sleep-related disturbances and physical inactivity are independently associated with obesity in adults. Int J Obes 31, 1713-21.

18) Mummery WK, Schofield G, Steele R, Eakin E, Brown WJ (2005) Occupational sitting time and overweight and obesity in Australian workers. Am J Prev Med 29, 91-7.

19) Access Economics. The economics costs of obesity (2006) Diabetes Australia. http://www.accesseconomics. com.au/publicationsreports/showreport.php?id=102. Accessed April 27, 2008.

20) National Heart Lung and Blood Institute www.nhlbisupport.com. Accessed April 27, 2008.

21) Sasaki T, Iwasaki K, Oka T, Hisanaga N (1999) Association of working hours with biological indices related to the cardiovascular system among engineers in a machinery manufacturing company. Ind Health 37, 457-63.

22) Sasaki T, Iwasaki K, Oka T, Hisanaga N, Ueda T, Fujiki $\mathrm{Y}$ (1999) Effect of working hours on cardiovascularautonomic nervous functions in engineers in a electronics manufacturing company. Ind Health 37, 55-61.

23) Bliwise DL (2005) Normal aging. N Kryger MK, Roth T, Dement WC (Eds.), 24-38, Principles and Practice of Sleep Medicine, Elsevier Saunders, Philadelphia.

24) Akerstedt T, Fredlund P, Gillberg M, Jansson B (2002) Work load and work hours in relation to disturbed sleep and fatigue in a representative sample. J Psychosom Res 53, 585-8.

25) Vgontzas AN, Lin H-M, Papaliaga M, Clhoun S, VelaBueno A, Chrousos GP, Bixler EO (2008) Short sleep duration and obesity: the role of emotional stress and sleep disturbances. Int J Obes 32, 801-9.

26) Dallman MF, Pecoraro NC, la Fleur SE (2005) Chronic stress and comfort foods: self-medication and abdominal obesity. Brain Behav Immun 19, 275-80.

27) Pecoraro NC, Reyes F, Gomez F, Bhargava A, Dallman MF (2004) Chronic stress promotes palatable feeding which reduces signs of stress: feedfoward and feedback effects of chronic stress. Endocrinology 145, 3754-62.

28) Taheri S, Lin L, Austin D, Young T, Mignot E (2004) Short sleep duration is associated with decreased leptin, elevated ghrelin, and increased body mass index. PLoS Med 1, 210-5.

29) Basner M, Fomberstein KM, Razavi RM, Banks S, William JH, Rosa RR, Dinges DF (2007) American time use survey: sleep time and its relationship to waking variables. Sleep 30, 1085-95.

30) Vorona RD, Winn MP, Babineau TW, Eng BP, Feldman HR (2005) Overweight and obese patients in a primary care population report less sleep than patients with a normal body mass index. Arch Int Med 165, 25-30. 
31) Peng C-YJ, Lee KL, Ingersoll GM (2002) An introduction to logistic regression and reporting. J Ed Res 96, 3-14.

32) Stevens J, Keil JE, Waid LR, Gazes PC (1990) Accuracy of current, 4-year, and 28-year old self-reported body weight in an elderly population. Am J Epidemiol 132, 1156-63.

33) Lockley SW, Skene DJ, Arendt J (1999) Comparison between subjective and actigraphic measurement of sleep and sleep rhythms. J Sleep Res 8, 175-83.

34) Van den Berg JF, Van Rooij FJA, Vos H, Tulen JHM, Hofman A, Miedema HME, Neven A, Tiemeier H (2008) Disagreement between subjective and actigraphic measures of sleep duration in a population-based study of elderly persons. J Sleep Res 17, 295-302. 